\title{
Research on Reduced-Order Modeling of Droop Controlled Inverter Suitable for Analysis of Transient Characteristics
}

\author{
TAN Yingjie ${ }^{1}$, XU Min ${ }^{1}$, GUO Zuogang ${ }^{1 *}$, LEI Jinyong ${ }^{1}$, YUAN Zhiyong ${ }^{1}$, ZHOU Changcheng $^{1}$ \\ ${ }^{1}$ Electric Power Research Institute, CSG, Guangzhou, Guangdong, 510663, China
}

\begin{abstract}
Under the background of a large number of converters connected to the power grid, the converter's weak overcurrent capability and poor anti-interference ability bring challenges and hidden dangers to the transient safe and stable operation of the power grid. It is urgent to carry out the transient characteristics analysis of the converter. Numerical simulation calculation is an important means to study transient characteristics. However, numerical simulation is time-consuming and poor in generality, which greatly limits the study of transient characteristics of systems with multiple converters. In this paper, the vertical control is the object, and the inverter reduced-order simplified model suitable for different transient stability problems is studied. First, a full-order model of the droop control inverter is established, and the singular perturbation method is used to complete the extraction of system characteristic roots and the analysis of participating factors. Then, according to the droop control of the inverter's power angle, voltage, and frequency transient response process, accuracy and rapidity are selected as the goals, and a reduced-order simplified model suitable for different transient stability analysis is obtained. The transient characteristic analysis of the numerical simulation of the converter system provides a reduced order reference.
\end{abstract}

\section{Introduction}

Converters have the problems of weak over-current capability and easy to be burned during transient processes such as short-circuit faults [1]. In addition, the converter lacks the physical inertia of the traditional RSG, resulting in poor anti-disturbance ability of the converter, and it is prone to serious stability problems when facing system disturbances, which brings challenges to system security [2]. In the US CERTS microgrid, a case of system collapse caused by high-power load switching has occurred [3]. In China, large-scale wind farms are offline due to stability issues, causing serious economic losses [4]. These safe operation problems are particularly prominent in the transient process. Therefore, it is of great value and significance to analyze the transient characteristics of the converter represented by the droop controled inverter when subjected to large disturbances such as short-circuit faults.

At present, there are two important methods to study the transient operation characteristics of the converter. One of the method is quantitative analysis represented by the Lyapunov energy function[5]. The advantage of this method is that it can give quantitative judgments on whether the converter is stable and the stability range in a certain state, but this method relies on constructing energy functions for quantitative analysis, and it is difficult to observe the key parameters of the system transient operation. At present, only systematic energy function construction methods exist for second-order nonlinear systems. Therefore, the application of this method to large-scale power grids needs to solve many technical problems. The other method to sutdy transient operation characteristics of converters is to observe numerical simulation results in time domain[6-7]. The advantages of this method are that there is no need to construct complex energy function, and the transient operation characteristics of the system can be observed in any initial state. Therefore, numerical simulation has become an important means of studying transient operation characteristics. However, with the increasing number of converters, the degree of the mathematical model shows a rapid upward trend, which brings a long time-consuming problem to the numerical simulation calculation [8]. In order to solve this problem, the mathematical model of converter with higher-order is reduced and simplified, and a reduced-order model suitable for different transient stability problems is obtained to effectively solve the problem.

In view of the characteristic of multi-time scale of the converter, a lot of researchs and explorations have been carried out on the order reduction and simplification of the converter on the time scale [9-10]. Existing research mainly studies the order reduction and simplification of models under small disturbance conditions, and lacks researchs on the order reduction and simplification for transient operation characteristics. In addition, the existing researches exclude system state variables mainly by their response speed for simlification. However, for

"Corresponding author's e-mail: guozg@esg.cn 
different transient time scales, such as transient voltage/frequency/power angle, the state variables that need to be considered are also inconsistent, hence, a certain part cannot be simply ignored.

In response to this problem, this paper takes the droop controlled inverter as the research object. Firstly, the state space model of the droop controlled inverter during normal operation and the detailed mathematical model of the droop inverter controlled by virtual impedance in case of failure are established. Because of the model has a higher order, the singular perturbation method is used to separate the fast and slow variables in the model to reduce the order. Subsequently, the influence of the simplified model on the simulation results of the droop controlled inverter when analyzing different transient characteristics is discussed. Finally, the optimal selection of model state variables and the recommended value of model order under different transient characteristics analysis conditions are summarized through the comparative study of numerical simulation, in order to speed up the model calculation speed while preserving the system dynamic process to the greatest extent.

\section{Transient model of droop controlled inverter}

\subsection{State space model of droop control}

The droop controlled inverter has the characteristics of plug and play, and has excellent grid friendliness. Generally, the core control of droop control has three parts: power control, voltage control and current control. A droop controlled inverter connected to infinite power grid is discussed in this paper, of which the main circuit is composed of three-phase bridge circuit. The output electric energy is transmitted to the power grid after filtering by LC filter loop. Figure 1 shows the topology of the system, where $R_{g}$ and $L_{g}$ are the resistance and inductance of the transmission line. For the convenience of the following discussion, the D-Q coordinate system is the grid voltage reference coordinate system and the $d-q$ coordinate system is the inverter coordinate syste. The power control part of inverter is divided into active power control and reactive power control loop.

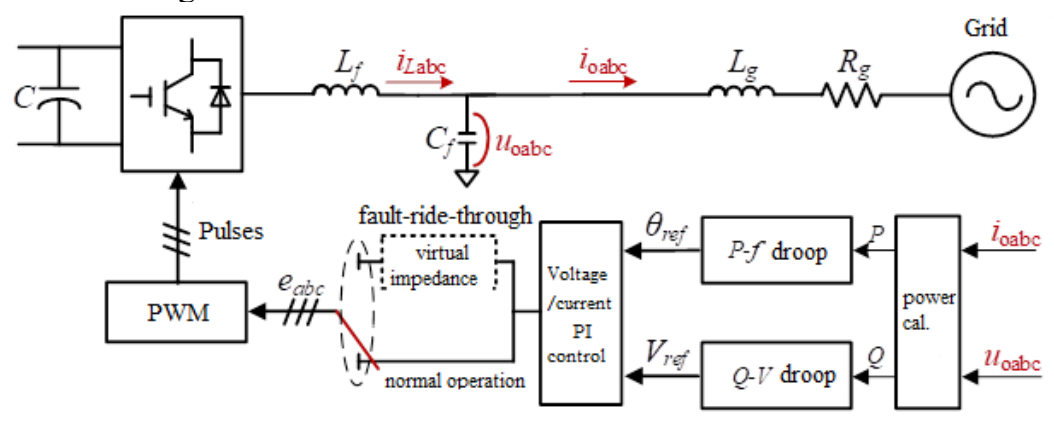

Figure 1. Topology of researched system

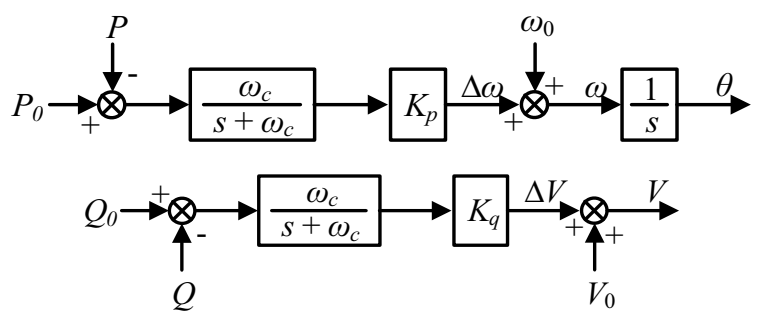

Figure 2. Structure of power control loops

According to Figure 2, the expression of the droop characteristic control link is:

$$
\begin{aligned}
& \omega=-D_{p}\left(P-P_{0}\right)+\omega_{0} \\
& u=-D_{q}\left(Q-Q_{0}\right)+u_{0}
\end{aligned}
$$

Where, $D_{p}, D_{q}$ are droop coefficients, $\omega_{0}$ and $u_{0}$ are rated angular velocity and voltage, respectively, $P_{0}$ and $Q_{0}$ are rated active and reactive power. What needs to be explained is that the active power $\mathrm{P}$ and reactive power $\mathrm{Q}$ obtained by real-time calculation have passed through the first order low-pass filtering link with turning frequency of $\omega_{c}$, namely:

$$
\begin{aligned}
& \dot{P}=\omega_{c}\left(1.5 u_{o d} i_{o d}+1.5 u_{o q} i_{o q}-P\right) \\
& \dot{Q}=\omega_{c}\left(1.5 u_{o q} i_{o d}-1.5 u_{o d} i_{o q}-Q\right)
\end{aligned}
$$

loop controller, and its state equation can be expressed as:

$$
\begin{aligned}
& i_{L d}^{r e f}=i_{o d}-\omega C_{f} u_{o q}+K_{v p}\left(u_{o d}^{r e f}-u_{o d}\right)+x_{1} \\
& i_{L q}^{r e f}=i_{o q}+\omega C_{f} u_{o d}+K_{v p}\left(u_{o q}^{r e f}-u_{o q}\right)+x_{2}
\end{aligned}
$$

Where, uod and uoq are obtained by coordinate transformation after voltage sampling at both ends of the capacitor, $K_{v p}$ is the voltage loop proportional control. $x_{I}$ and $x_{2}$ are:

$$
\begin{gathered}
\left.\dot{x}_{1}=K_{v i} L-D_{q}\left(Q-Q_{0}\right)+u_{0}-u_{o d}\right] \\
\dot{x}_{2}=K_{v i}\left(0-u_{o q}\right)
\end{gathered}
$$

Where, $K_{v i}$ is the voltage loop integral control gain.

Similarly, the state equations of the current loop are:

$$
u_{d}=-\omega L_{f} i_{L q}+K_{c p}\left(i_{L d}^{r e f}-i_{L d}\right)+x_{3}
$$




$$
u_{q}=\omega L_{f} i_{L d}+K_{c p}\left(i_{L q}^{r e f}-i_{L q}\right)+x_{4}
$$

Where, $K_{c p}$ is the voltage loop proportional control gain. $x_{3}, x_{4}$ are:

$$
\begin{aligned}
& \dot{x}_{3}=K_{c i}\left(i_{L d}^{r e f}-i_{L d}\right) \\
& \dot{x}_{4}=K_{c i}\left(i_{L q}^{r e f}-i_{L q}\right)
\end{aligned}
$$

The state equations of filter element and line impedance are as follows:

$$
\begin{gathered}
L_{f} \frac{d i_{L d}}{d t}=u_{L d}-u_{o d}+\omega L_{f} i_{L q} \\
L_{f} \frac{d i_{L q}}{d t}=u_{L q}-u_{o q}-\omega L_{f} i_{L d} \\
C_{f} \frac{d u_{o d}}{d t}=i_{L d}-i_{o d}+\omega C_{f} u_{o q} \\
C_{f} \frac{d u_{o q}}{d t}=i_{L q}-i_{o q}-\omega C_{f} u_{o d} \\
L_{f} \frac{d i_{o d}}{d t}=u_{o d}-i_{o d} R_{g}+\omega L_{g} i_{o q}-u_{g d} \\
L_{f} \frac{d i_{o q}}{d t}=u_{o q}-i_{o q} R_{g}-\omega L_{g} i_{o d}-u_{g q}
\end{gathered}
$$

The angle between the coordinate system dq of the inverter and the coordinate system DQ of the power grid side is $\delta$ as follows:

$$
\frac{d \delta}{d t}=\omega-\omega_{0}
$$

It can be seen that the complete model of droop control consists of equations (1) to (19), where have 13 state variables, namely $\left[\delta, P, Q, i_{o d}, i_{o q}, u_{o d}, u_{o q}, i_{L d}, i_{L q}, x_{l}\right.$, $\left.x_{2}, x_{3}, x_{4}\right]$. Therefore, it is necessary to find the dominant variable of transient angle instability by means of singular perturbation method.

\subsection{Current limiting control of droop controlled inverter during fault}

In order to ensure the safe operation of the inverter during the fault, the virtual impedance can be added to the control loop to limit the current. For the sake of simplicity and versatility, this article adopts direct access virtual impedance $R_{v}$ and $L_{v}$ with fixed value. During the fault process, the controller modulation wave generation switch in Figure 1 switches from the normal operation end to the fault ride-through end. The mathematical expressions (17)-(18) of the droop controlled inverter with the added virtual impedance become (whether the virtual impedance be directly added to the line).

$$
\begin{aligned}
& L_{f} \frac{d i_{o d}}{d t}=u_{o d}-i_{o d}\left(R_{g}+R_{v}\right)+\omega\left(L_{g}+L_{v}\right) i_{o q}-u_{g d} \\
& L_{f} \frac{d i_{o q}}{d t}=u_{o q}-i_{o q}\left(R_{g}+R_{v}\right)-\omega\left(L_{g}+L_{v}\right) i_{o d}-u_{g q}
\end{aligned}
$$

As can be seen, the introduction of virtual impedance will not change the order of the model, but will weaken the power grid to some extent. The equivalent impedance of the model used in this paper is $0.3+\mathrm{j} 1.257 \Omega$ during the fault period. The mathematical model of the droop controlled inverter is still 13 since the introduced virtual impedance control does not introduce a new order during the fault.

\section{Reduction and simplification of droop controlled inverter model based on singular perturbation method}

There are different time scales in these control links, as shown in Figure 3. From the time scale, we can choose the reduced-order model according to different research objects. Therefore, this paper uses singular perturbation method to reduce the order of droop controlled inverter.

According to the singular perturbation method, the dynamic model of the system discussed in this paper can be expressed abstractly as:

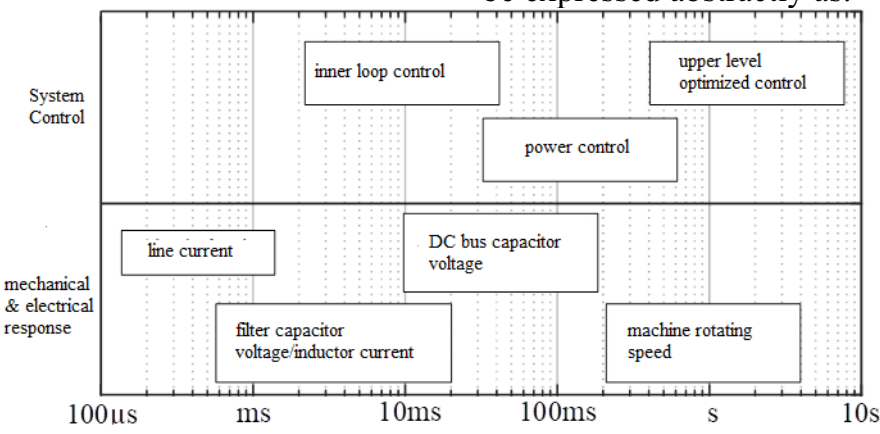

Figure 3. Time scales of microgrid's dynamic characteristics

$$
\begin{aligned}
\dot{x} & =f(t, x, z, \varepsilon) \\
\varepsilon \dot{z} & =g(t, x, z, \varepsilon)
\end{aligned}
$$

Among them, $\varepsilon$ is the disturbance parameter matrix reflecting the characteristics of the system, diag $\left\{\varepsilon_{l}\right.$, $\left.\varepsilon_{2}, \ldots, \varepsilon_{m}\right\} . \mathrm{X}$ and $\mathrm{z}$ represent fast state variables and slow state variables, respectively. The differential equation (23) degenerates into an algebraic equation when $\varepsilon$ equal to 0 . Substituting this algebraic equation to formula (22), the effect of fast state variables on the system can be eliminated. Thus, only the slow state vector is left in the equation, and the model is reduced. From formula (1) to formula (19), the equilibrium point of the system and the eigenvalue of the linear state matrix at the running point can be solved by MATLAB/Simulink. Figure 4 shows the 
complete eigenvalue distribution of the system studied in this paper at the equilibrium point.

According to the theory of small signal analysis, the eigenvalue can describe the mode of dynamic response of the system. For a pair of conjugate eigenvalues $\lambda=\sigma \pm j \omega$, the pair of eigenvalues can reflect the corresponding oscillation mode $\mathrm{e}^{\sigma t} \sin (\omega t+\theta)$ of a system, so the oscillation damping of the system can be obtained from the real and imaginary parts of the eigenvalues, as follows:

$$
\xi=\frac{-\sigma}{\sqrt{\sigma^{2}+\omega^{2}}}
$$

The real part of eigenvalue $\sigma$ reflects the speed of attenuation. In Figure4, the distribution of eigenvalues observed varies from -1500 to -4 , which indicates that different modes of the system have obvious differences in dynamic response rate, so the model can be separated and simplified by the fast and slow variables.

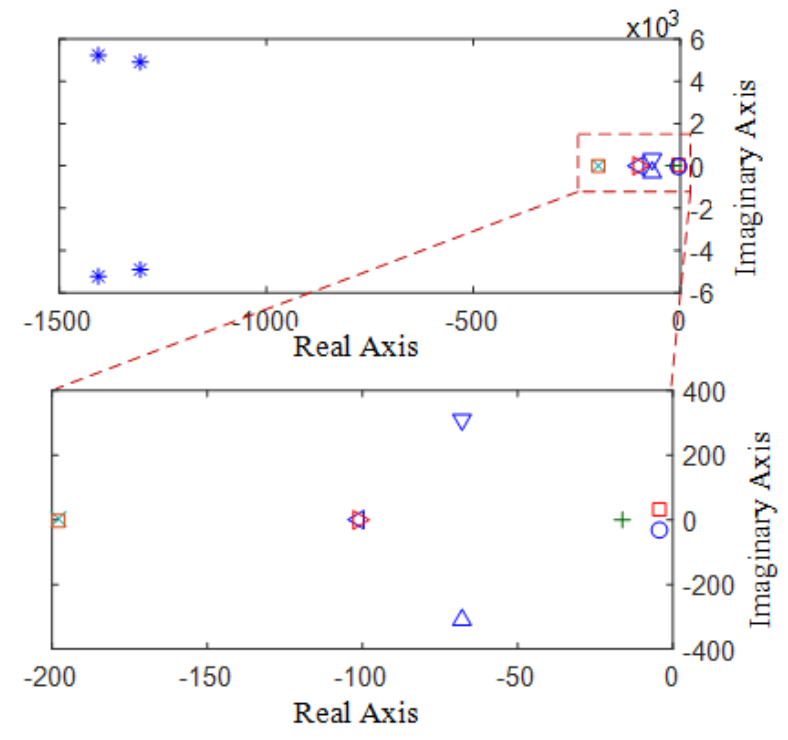

Figure 4. Eigenvalue spectrum of the system state matrix

Take the voltage and current loop as an example to briefly describe the reduction process. The voltage-current loop contains four differential equations, as shown in formulas (7), (8), (11), (12), respectively. Reworded to singular perturbation, there are:

$$
\begin{gathered}
\frac{1}{K_{v i}} \frac{d x_{1}}{d t}=U_{n}-D_{q} Q-u_{o d} \\
\frac{1}{K_{v i}} \frac{d x_{2}}{d t}=0-u_{o q} \\
\frac{1}{K_{c i}} \frac{d x_{3}}{d t}=i_{L d}^{r e f}-i_{L d} \\
\frac{1}{K_{c i}} \frac{d x_{4}}{d t}=i_{L q}^{r e f}-i_{L q}
\end{gathered}
$$

In these 4 equations, the perturbation parameter $\varepsilon$ equal to $\left\{1 / K_{v i}, 1 / K_{v i}, 1 / K_{c i}, 1 / K_{c i}\right\}$. The differential equation is simplified to an algebraic equation if the perturbation parameter is zero.

$$
u_{\text {od }}=U_{n}-D_{q} Q
$$

$$
\begin{array}{r}
u_{o q}=0 \\
i_{L d}=i_{L d}^{r e f} \\
i_{L q}=i_{L q}^{r e f}
\end{array}
$$

So far, the reduction of voltage and current double closed loop is completed.

\section{4 selection of characteristics of droop controlled inverter simplified model}

This paper uses the running time of the 13th order model calculation as the benchmark of simplified model calculating speed. The ratio of the time difference between calculating time of the simplified model and calculating time of the 13 th order model to calculating time of the 13th order model in each scenario is used as the criterion of rapidity.

For accuracy, which is the ratio of the maximum value of the difference between the simplified model and the full order model curve in the simulation process to the value of the full order model at this moment, as shown in formula (33).

$$
\text { errorrate }=\frac{\left|v a l_{13^{t h}}(t)-v a l_{x^{t h}}(t)\right|}{v a l_{13^{t h}}(t)}
$$

The time scale of transient work angle stability problems is usually from $100 \mathrm{~ms}$ to s levels. Therefore, consider building mathematical model $X_{a l}=[P, Q, \delta]$ with third-order and $X_{a 2}=\left[P, Q, \delta, i_{o d}, i_{o q}\right]$ with 5 th order. Building a numerical simulation model in the MATLAB/Simulink, and the accuracy of the two simplified models in describing the power angle dynamics in transient process is compared.

Figure 5- 7 shows the waveform comparison between the simplified model and the full order model in calculating the characteristics of the transient power angle. What needs to be explained is that the power angle here refers to the virtual power angle, which is defined as the angle between the droop controlled inverter rotating $\mathrm{dq}$ coordinate system and the grid side rotating DQ coordinate system. Setting the three-phase grounding short circuit fault on the power grid side, as a result, the voltage of the power network drops suddenly. It can be seen that both of the third and fifth order models can fit the dynamic response of the full order model well in the early stage of failure. Especially in the power angle curve which is more concerned in the transient power angle characteristic analysis, the simplified model and the full order model are almost identical at the initial stage of the fault. Although the fitting of the 3rd order model to the active power curve loses some fast adjustment process caused by the voltage and current loop, the overall trend is consistent with the full and 5th order models. 


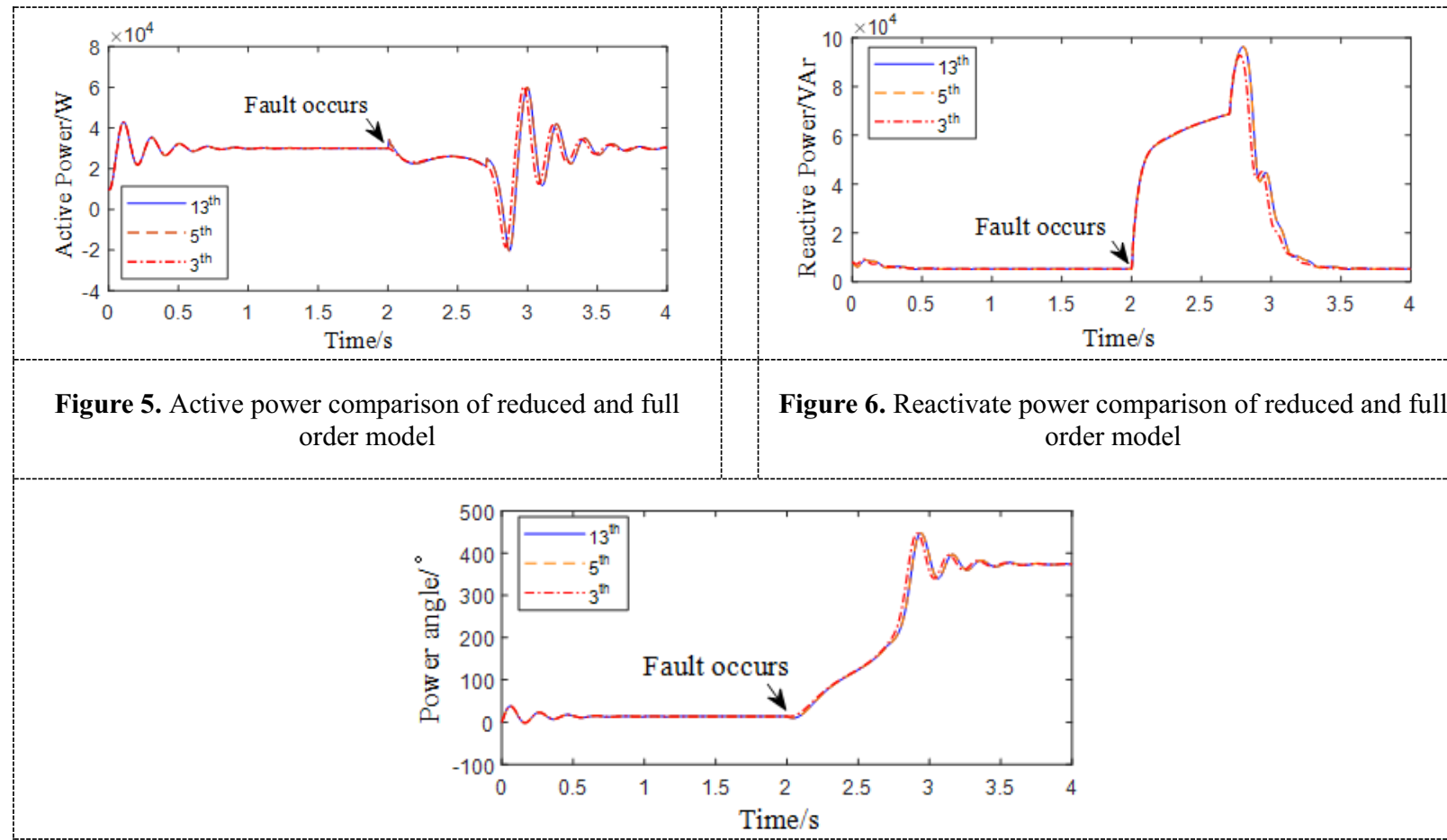

Figure 7. Power angle comparison of reduced and full order model

However, the dynamic of the third order model is quite different from that of the fifth order and the full order model in the late stage of the fault and after the fault removal, which is shown as the advance of the fitting curve in Figure 5- 7. This means that when the third order model is used to analyze the stability of the system, a more conservative conclusion will be obtained, which will inevitably lead to the reduction of the boundary when depict the boundary of the stable domain. The calculation time of simplified model and full order model is shown in Table 1.

As seen in the simulation waveforms, the simplified model can roughly fit the change of the full order model.
However, all the simplified models lose the dynamic adjustment process of partial output voltage amplitude, in the early stage of failure. In the later stage of simulation, the fitting effect of simplified model is better, and the difference between simplified models is tiny. From the point of view of stability analysis, the boundary difference between the simplified model and the full order model will not be very large, and the conservatism o estimated by the simplified model will be acceptable.

Similarly, the calculation time for transient voltage characteristic analysis and frequency characteristic analysis are shown in Table 1.

Table 1. Comparison of Reduced-Order and Full-Order Models

\begin{tabular}{|c|c|c|c|c|c|}
\hline Model category & transient process & order & $\begin{array}{l}\text { Speed lift rate of } \\
\text { simplified model }\end{array}$ & $\begin{array}{l}\text { Simplified } \\
\text { model error } \\
\text { rate }\end{array}$ & $\begin{array}{l}\text { Conclusion of } \\
\text { optimal selection }\end{array}$ \\
\hline \multirow[t]{2}{*}{ full order model } & -- & 13 & -- & -- & \\
\hline & Transient power angle & 5 & $37.61 \%$ & $0.061 \%$ & $\sqrt{ }$ \\
\hline \multirow{9}{*}{$\begin{array}{l}\text { simplified } \\
\text { models }\end{array}$} & \multirow{5}{*}{$\begin{array}{l}\text { transient } \\
\text { voltage }\end{array}$} & 3 & $63.49 \%$ & $1.630 \%$ & \\
\hline & & 7 & $14.92 \%$ & $1.206 \%$ & \\
\hline & & 5 & $37.41 \%$ & $1.211 \%$ & \\
\hline & & 3 & $64.91 \%$ & $1.211 \%$ & $\sqrt{ }$ \\
\hline & & 7 & $14.92 \%$ & $3.276 \%$ & \\
\hline & \multirow{2}{*}{$\begin{array}{l}\text { Resistive } \\
\text { circuit }\end{array}$} & 5 & $37.41 \%$ & $3.283 \%$ & \\
\hline & & 3 & $64.91 \%$ & $3.286 \%$ & $\sqrt{ }$ \\
\hline & \multirow{2}{*}{ transient frequency } & 5 & $21.69 \%$ & $0.007 \%$ & \\
\hline & & 3 & $64.27 \%$ & $0.148 \%$ & $\sqrt{ }$ \\
\hline
\end{tabular}


The purpose of the model reduction simplification is to keep the whole order model dynamic as much as possible while maximizing the calculation difficulty and improving the calculation speed and efficiency. Therefore, on the basis of the above discussion, combined with the calculation time of each simplified model in Table 1, the most suitable simplified model for characteristic analysis of different transient processes can be obtained.

\section{Conclusion}

(1) For the analysis of transient characteristics in different time scales, the corresponding eigenvalues of the system should be selected to reduce the order;

(2) For the analysis of transient power angle characteristics, the fifth order model can fit the dynamic process of the full order model in a long time, while the simulation speed is fast. The stable domain of the 5 th order model is less conservative than the 3rd order model;

(3) For the analysis of transient voltage characteristics, two scenarios of inductive circuit and resistive circuit with small resistance are considered because the ratio of resistance to inductance will determine the coupling degree of active and reactive power. The 3 rd order simplified model composed of $\left[i_{o d}\right.$, $\left.i_{o q}, Q\right]$ which can quickly calculate the dynamic changes of the system in a short time is picked because the 3rd and 5th order models have similar error rates in the case of resistive and inductive lines. For large resistance, just follow this article to calculate and select, which will not be repeated;

(4) For the analysis of the transient frequency characteristics, the third order simplified model composed of $[P, Q, \delta]$ can not only satisfy the requirement of quick calculation, but also can fit the dynamic response of the system for a long time.

\section{Acknowledgments}

This work was supported by the Science \& Technology Project of China Southern Power Grid (ZBKJXM20180080).

\section{References}

1. Shuai Z, Huang W, Shen C, et al. Characteristics and restraining method of fast transient inrush fault currents in synchronverters[J]. IEEE Transactions on Industrial Electronics, 2017, 64(9): 7487-7497.

2. Shuai Z, Shen $C$, Yin $X$, et al. Transient angle stability of virtual synchronous generators using Lyapunov's direct method, IEEE Trans. Smart Grid, vol. 10, no. 4, pp. 4648-4661, Jul. 2019.

3. Paquette A, Reno M, Harley R, and Divan D, Sharing transient loads: causes of unequal transient load sharing in islanded microgrid operation, IEEE Ind. Applicat. Mag., vol. 20, no. 2, pp. 23-34, Dec.2013.

4. Ö. Göksu, R. Teodorescu, C. L. Bak, F. Iov, and P. C.
Kjær, Instability of wind turbine converters during current injection to low voltage grid faults and PLL frequency-based stability solution," IEEE Trans. Power Syst., vol. 29, no. 4, pp. 1683-1691, Jul. 2014.

5. M. Kabalan, P. Singh, and D. Niebur, Large signal lyapunov-based stability studies in microgrids: a review, IEEE Trans. Smart Grid, vol.8, no. 5, pp. 2287-2295, Sep. 2017.

6. Peng Y, Shuai Z, Shen J, c Reduced order modeling method of inverter-based microgrid for stability analy-sis[C]//2017 IEEE Applied Power Electronics Conference and Exposition (APEC). IEEE, 2017: 3470-3474.

7. Kabalan M, Singh P, Niebur D. Nonlinear Lyapunov stability analysis of seven models of a DC/AC droop controlled inverter connected to an infinite bus[J]. IEEE Transactions on Smart Grid, 2017.

8. Shuai Z, Peng Y, Yin X, et al.Dynamic Equivalent Modeling for Multi-Microgrid Based on Structure Preservation Method, IEEE Trans. Smart Grid, vol. 10, no. 4, pp. 3929-3942, July 2019.

9. Shuai Z, Peng Y, Yin X, et al. Guerrero and Z. J. Shen, Parameter Stability Region Analysis of Islanded Microgrid Based on Bifurcation Theory, IEEE Transactions on Smart Grid, vol. 10, no. 6, pp. 6580-6591, Nov. 2019.

10. Shuai Z, Peng Y, Guerrero J. M, et al. Transient Response Analysis of Inverter-Based Microgrids Under Unbalanced Conditions Using a Dynamic Phasor Model, IEEE Transactions on Industrial Electronics, vol. 66, no. 4, pp. 2868-2879, April 2019. 